\title{
Responders vs Non-Responders to Music Therapy
}

\section{P.Barathi*}

During music administration to patients, I have always been confronted with the interesting phenomenon of coming across patients who respond well to music therapy on the very first session itself, these individuals gradually exhibiting signs of improvement after a duration of 3-4 weeks, as opposed to others who either show no response at all or become restless even before 10 minutes of listening, and it is not surprising that they fail to show any improvement even if they are made to listen regularly. I found that this phenomenon was repeated, even if the patients were made to choose the music according to their own preferences.

\section{WHAT COULD BE THE REASONS BEHIND THESE UNIQUE VARIATIONS AMONG INDIVIDUALS REGARDING MUSIC STIMULATION?}

1. Musical Intelligence- The "Expression" in Music as Interpreted or Felt by a Performer/ Audience :

According to Dr. Howard Gardner there are 7 Emotional Intelligences, viz., verbal( the ability to use words), visual( the ability to see things in your mind), physical( the ability to use your body well), musical (the ability to understand and use music), mathematical/logical( the ability to apply logic), intrapersonal( the ability to understand thoughts and feelings), and interpersonal (the ability to relate well to others).

The common issue faced by researchers is that it is hard to classify music based on its emotional content. The emotional content of music is difficult to discuss since it is a "subjective" experience, meaning that each listener or performer may construct a slightly different personal experience, even for the same music. It is important to consider the fundamental issue between music and human perception: the possibility of educating or enhancing our emotional responses to music.

Many people take it for granted that listening to music requires no skill, but it is vital to remember that not only musicians but also listeners need to improve the ability to understand music in order to fully appreciate it. Differences among interpretations of music do exist, our perception of emotion from music varying, depending on varying situations and individual differences in perception- not everybody associates the same musical features with the same emotion.

According to Feieraband,JM(1995), individuals with high music intelligence "think music" with greater clarity and are affected more deeply by music, in an aesthetic sense, than those with less music intelligence.

\section{Musical INTELligenCe AND ReSPONSE to Music TherAPY}

Recent insights about the psychological development of musical thinking have revealed that musical intelligence is nurtured by the children at a very early age. Since synaptic connections are at their peak production at the age of 2 , music stimulation should begin immediately at birth in order to preserve musical intelligence, because it starts declining prior to the age of 5. According to researchers, a child stores a mass of musical impressions well before commencing school education, and what a child has heard in his first six years of life cannot be eradicated later. Therefore, it is of utmost importance to stimulate musical intelligence well before the child starts entering school life.

\section{HOW TO STIMULATE MUSICAL INTELLIGENCE?}

To develop musical intelligence, one must develop neuronal pathways for musical thinking early in life. If the musical mind is engaged in early stimulation through music listening or playing, we establish neuronal pathways that will understand and perceive music from this intellectual framework, since the synapses are formed, strengthened, and maintained by interaction with repeated experience, as a result of the information being processed through certain conditioning. Appropriate stimulation of musical intelligence from the earliest experiences is necessary, if the pathways are to be built to understand musical phenomena from a musical perspective. Once the pathways for understanding

\footnotetext{
* Dr. P.Barathi, M.D.,

Prof of Physiology, Music Therapist

e-mail : iyer.bharthi@gmail.com
} 
musical or any other phenomenon for that matter are established, it is difficult to reshape the mind to perceive that same phenomenon from a different perspective.

"It is much more difficult to reorganize a brain than it is to organize it in the first place. Organization inhibits reorganization. Carving out neuronal tracks for certain types of learning is best accomplished when the synapses for that particular skill are malleable, before they harden up around certain types of responses." Jane M. Healy.

Musical intelligence can be demonstrated by the fact that individuals who learn to play an instrument through ready notation- without appropriate musical skills such as singing and moving with music- engage only their logical/ mathematical intelligence and would most likely have later difficulty to play by ear. Edwin Gordon has suggested that music intelligence is determined by one's ability to "think" music, or in his words to "audiate" music. The better one is in creating music inside one's head, the better developed is one's potential to succeed in real musical understanding and appreciation.

The emotional content of music is difficult to discuss since it is a subjective" experience, meaning that each listener or performer may construct a slightly different personal experience, even for the same music.

\section{The Psycho- NeUro- Endocrinologic Responses to Music Stimulation}

It is well known that individuals and cultures identify emotionally with different musical stylings. What is stimulating for one may induce a neutral response to another. The reason could be attributed to the psycho-neuro-endocrine effects of music in the human brain and body which provide direct links between music stimulation, activations in the brain during music processing and emotional response at both physiological and subjective levels. Music emotion research at the hormonal levels might yield more information regarding the relationship between perceived and felt emotions during music stimulation. In other words, by understanding how music affects the endocrine system, we will increase our knowledge of how music affects emotions. Ultimately, research in psycho-neuro-immunology should contribute to answering questions about how musical behaviours function as psychological components in processes related to wellbeing and health ( Kreutz et al., 2012).

According to Blood \&Zatorre (2001 ), intensely pleasurable responses to music are associated with activity in brain regions implicated with reward and emotion. Pleasurable music invokes emotions releasing the reward hormone dopamine, in correlation with the "chills" running up and down the spine during intense peak responses. It has already been established that strongly felt emotions could be rewarding in themselves in the absence of a physically tangible reward or a specific functional goal, and it is amazing to find that there is also an anticipatory release of dopamine to an aesthetic stimulus- a stimulus that is abstract, complex and not concrete (Salimpoor et al.,2009).

Are there any other factors that play a role in healing the signs and symptoms of the disease process in those who respond well to music therapy?

\section{THE INTRICATE BIOLOGICAL TIME STRUCTURES IN HUMANS :}

Overview of the favored periodic duration (frequency ranges) of the rhythmic functions in humans. The scale of periodic duration is divided logarithmically. The stable frequency ranges of the spontaneous rhythms are specially emphasized.

Figure : 1

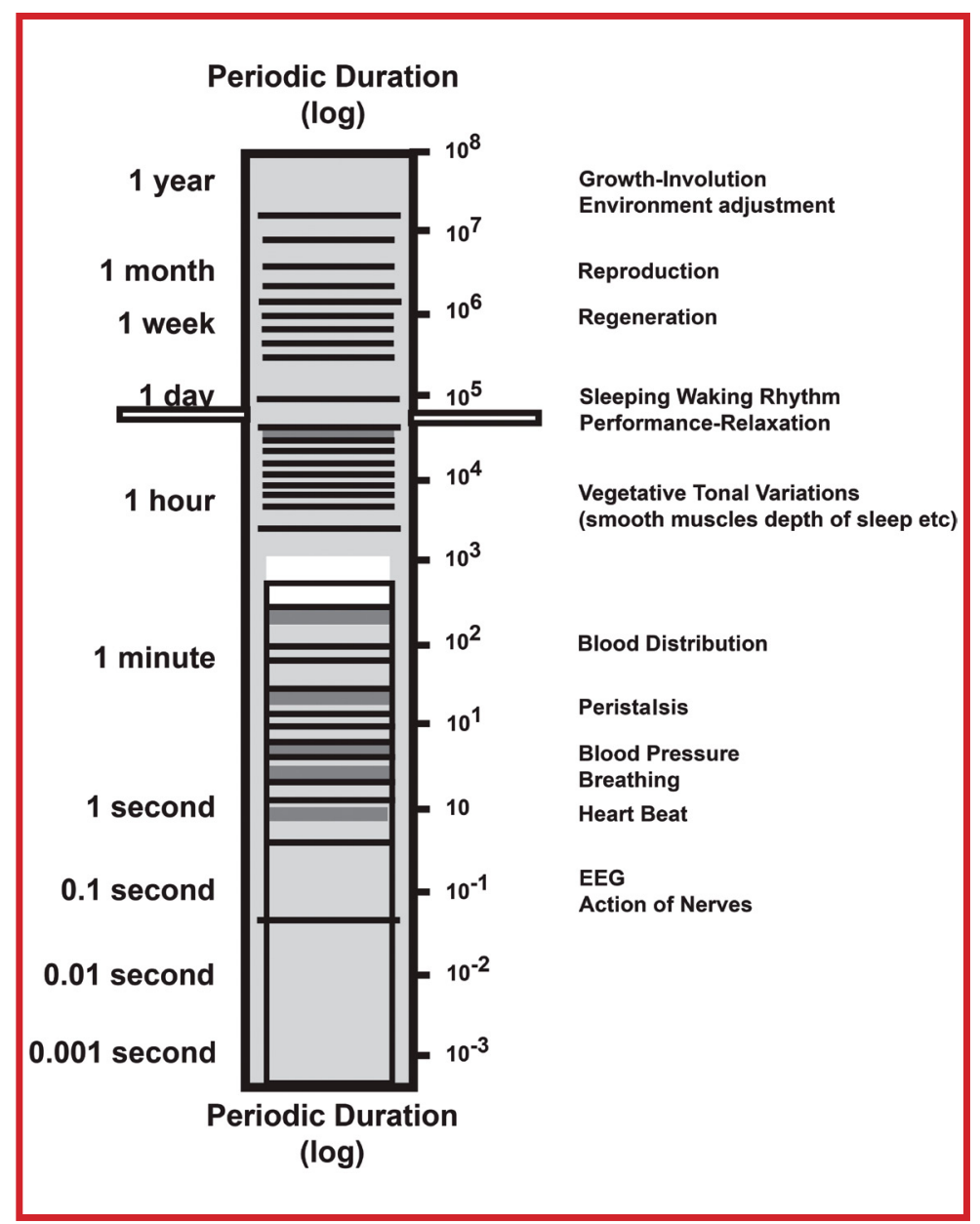


The results of modern chronobiology and chronomedicine have shown that the human organism does not only have a complicated interior design in terms of space structure, but also has a foundation based on an intricate time design, being composed of numerous rhythmical time structures_the intricate biological time structures in humans (Vide Fig:1). Figure $: 1$ shows a spectrum of the main types of rhythmical functions, arranged logarithmically according to the periodic duration. It includes approximately 2 into 12 octaves, from approximately one millisecond to an order of one year. With an increasing periodic duration, the complexity of the rhythms grow, from the cellular rhythms via the tissue and organ rhythms towards the rhythms of more complexity, finally including the entire organism and even the population rhythms- a hierarchical structure existing and determining the way in which the longer wave rhythms include the shorter ones. This illustrates that there are structures in humans which follow musical conformities to a natural law. The whole of the human organism participates as a moving entity, as a sensory being, and as an intellectual receptor in experiencing music. This could be the ultimate achievement for music in entraining the organism and shaping regularities from irregularities. In this way music therapy becomes an autonomous and not merely operative discipline, in other words a therapeutic application of music.

\section{REFERENCES}

1. Blood AJ and Zatorre RJ (2001). Intensely pleasurable responses to music correlate with activity in brain regions implicated in reward and emotion. Proceedings of the National Academy of Sciences of The United States of America; vol. 98 ( 20) 11818-11823.

2. Edwin Gordon (1968).A Study of the Efficacy of General Intelligence and Musical Aptitude Tests in Predicting Achievement in Music. In Bulletin of the Council for Research in Music Education.No. 13, pp. 40-45.University of Illinois Press

3. Feierabend, J.M.(1995). Music and Intelligence in the Early Years. The Hartt School University of Hartford From Early Childhood Connections.

4. Gardner, Howard (1983; 1993) Frames of Mind: The theory of multiple intelligences, New York: Basic Book 5. Gutenbrunner,C., Moog,R, and Hildebrandt,G, (1993). In Chronobiology \& Chronomedicine. Peter Lang GmbH Kreutz G., Murcia CQ and Bongard,S (2012). Psychoneuroendocrine Research on Music and Health: An Overview In Music, Health, \& Wellbeing. MacDonald R, Kreutz,G,and LauraMitchell (Eds). Oxford University Press. 6.Salimpoor VN, Benovoy M, Longo G, Cooperstock JR, Zatorre RJ (2009) The Rewarding Aspects of Music Listening 7. Are Related to Degree of Emotional Arousal. PLoS ONE 4(10): e7487.

artistic results. 